Résumés des conférences et travaux

\title{
Philologie et historiographie du Caucase chrétien
}

Jean-Pierre Mahé

\section{(2) OpenEdition \\ Journals}

\section{Édition électronique}

URL : https://journals.openedition.org/ashp/4140

DOI : $10.4000 /$ ashp. 4140

ISSN : 1969-6310

\section{Éditeur}

Publications de l'École Pratique des Hautes Études

\section{Édition imprimée}

Date de publication : 1 septembre 2021

Pagination : 55-66

ISSN : 0766-0677

\section{Référence électronique}

Jean-Pierre Mahé, "Philologie et historiographie du Caucase chrétien », Annuaire de l'École pratique des hautes études (EPHE), Section des sciences historiques et philologiques [En ligne], 152 | 2021, mis en ligne le 14 juin 2021, consulté le 16 juin 2022. URL : http://journals.openedition.org/ashp/4140 ; DOI : https://doi.org/10.4000/ashp.4140 


\title{
PHILOLOGIE ET HISTORIOGR APHIE DU CAUCASE CHRÉTIEN
}

\author{
Directeur d'études : M. Jean-Pierre MAHÉ, \\ membre de l'Institut
}

Programme de l'année 2019-2020 : I. Histoire primitive du Kartli et Listes royales dans la Conversion de la Géorgie. - II. Le roman des Saints Traducteurs et l'enseignement de la philosophie dans l'Arménie des $V I^{e}-X I^{e}$ siècles.

I. Tel que nous le lisons dans l'édition des Monuments hagiographiques de la Géorgie (MHG), par Ilia Abuladze, l'ensemble intitulé Conversion du Kartli (CK) réunit sans aucun doute plusieurs unités narratives d'origines et de dates diverses. Mais cela n'implique pourtant pas que l'œuvre manque d'unité conceptuelle ou littéraire. Au contraire, à chaque pas, on découvre la solidarité rédactionnelle des parties et du tout.

Le premier chapitre se détache de toute la suite. Il contient un survol du destin historique de la Géorgie, depuis la fondation du royaume ibère $\sim \mathrm{III}^{\mathrm{e}}$ siècle jusqu'à la campagne victorieuse d'Héraclius en 630. Au cœur de ce parcours s'inscrit un bref récit chronologique de la prédication de sainte Nino et de son apostolat au Kartli.

Cette narration est marquée, au début, au milieu et à la fin, par l'évocation de trois empereurs, Alexandre, Constantin et Héraclius. Nous l'appellerons donc, par commodité, «Chronique des trois empereurs » (C3E). Comme elle ne contient aucune allusion aux Arabes, il n'y a pas d'inconvénient à supposer qu'elle remonte à la première moitié du VII ${ }^{\mathrm{e}}$ siècle, au lendemain des victoires byzantines contre les Sassanides et à la veille des premières incursions califales dans le Caucase, en 639. Toutefois, comme les manuscrits qui nous la transmettent sont datables des $\mathrm{IX}^{\mathrm{e}}-\mathrm{XIII}{ }^{\mathrm{e}}$ siècles, certains auteurs ont estimé qu'elle ne saurait être antérieure au viII ${ }^{\mathrm{e}}$ siècle.

Les chapitres suivants CK II-XV ont un rapport évident avec ce qui précède. Plusieurs d'entre eux éclairent ou explicitent les principaux épisodes de la carrière de Nino en Géorgie. Ces chapitres contiennent aussi un récit tout à fait nouveau sur les origines familiales de Nino et sur sa jeunesse, partagée entre la Cappadoce et la Terre Sainte, ainsi que sur les circonstances, romanesques ou providentielles, de sa venue dans le Caucase.

Le tout est présenté dans un ordre non chronologique, comme un Dossier sur Nino réunissant plusieurs témoignages masculins et féminins, y compris les mémoires de la sainte elle-même. Les indices internes manquent pour savoir où et quand ce Dossier a été constitué, ou purement et simplement composé comme une œuvre littéraire. Quant aux indices externes, la liste des catholicos-patriarches de Géorgie qui sépare, dans le manuscrit NSin50 et dans le manuscrit de Č'eliš, la "Chronique des trois empereurs » $(\mathrm{C} 3 \mathrm{E})$ du Dossier sur Nino, a été tenue à jour jusqu'au début du $\mathrm{X}^{\mathrm{e}}$ siècle. Par conséquent, le modèle, aujourd'hui perdu de NSin50 et de Č'eliš, remonte au plus tard au IX ${ }^{\mathrm{e}}$ siècle. 
À cette époque, depuis 883, Adarnase Bagrat'ioni, curopalate du T'ao K'lardžeti, en Géorgie pontique, s'efforçait d'ériger sa principauté en royaume, ce qui advint en 916. En 971, fut copié au monastère de Šat'berd, dans ce nouveau royaume, un manuscrit réunissant $\mathrm{C} 3 \mathrm{E}$ au Dossier sur Nino. Il semble donc vraisemblable que ce dernier écrit fut composé au IX ${ }^{\mathrm{e}}$ siècle, soit au T'ao K'lardžeti, soit plus vraisemblablement dans un monastère géorgien de Jérusalem, étant donné la familiarité de l'auteur avec les lieux saints.

Cette hypothèse sur les deux phases de composition de la Conversion $d u$ Kartli (selon nous aux $\mathrm{VII}^{\mathrm{e}}$ et $\mathrm{IX}^{\mathrm{e}}$ siècles) est confirmée par la cohérence rédactionnelle de chacune de ces deux composantes. Nous le montrerons ci-dessous pour les deux premières unités narratives de $\mathrm{C} 3 \mathrm{E}$, qu'on appelle conventionnellement « Histoire primitive du Kartli » (CK I, 1) et « Liste des rois païens » (CK I, 2).

Uniquement préservée dans le codex de Šat'berd, cette liste semble d'abord se présenter comme un simple squelette, énumérant 27 noms, depuis Azo, premier roi du Kartli installé par Alexandre, jusqu'à Mirian, converti au christianisme par sainte Nino. Certains noms sont accompagnés de maigres commentaires indiquant les idoles érigées par les monarques et la part qu'ils ont prise à la construction des villes et forteresses de Mcxeta ou des alentours. D'autres noms sont cités, sans plus. Dans l'ensemble, on ne trouve aucune indication sur les différentes dynasties, la durée des règnes, ou la chronologie en général.

Si l'on compare cet inventaire décharné aux notices souvent bien fournies ou même aux récits étoffés que Leont'i Mroveli (Léonce, archevêque de Ruisi) consacre à chacun des monarques dans la « Vie des rois du Kartli»(VK), on est d'abord fortement tenté de supposer que l'auteur de la Conversion du Kartli s'est limité à désosser l'œuvre de Leont'i pour n'en garder qu'un schéma minimal. Si d'aventure on retenait cette hypothèse, il faudrait renoncer à dater du $\mathrm{VII}^{\mathrm{e}}$ siècle $\mathrm{C} 3 \mathrm{E}$, puisque Leont'i Mroveli est souvent considéré comme un auteur $\mathrm{du}_{\mathrm{XI}}{ }^{\mathrm{e}}$ siècle, remaniant éventuellement des sources du VIII ${ }^{\mathrm{e}}$ siècle. Cependant il y a, croyons-nous, deux bonnes raisons de soutenir que, pour la succession des règnes, VK et CK recourent, indépendamment, à une source commune.

Tout d'abord, certaines divergences entre les deux listes de rois sont très révélatrices. Ainsi, dans CK, Mirian, le premier roi chrétien, est le $27^{\circ}$ monarque. Dans VK il est le 24e. L'écart s'explique ainsi :

$1^{\circ} \mathrm{VK}$ ne compte pas Azon comme un vrai roi dans sa liste; instauré par Alexandre, il le considère comme un tyran illégitime.

$2^{\circ} \mathrm{Au}$ moment de la diarchie (de 58 à 106), Leont'i mentionne ( $\left.\mathrm{n}^{\circ} 15\right)$ une seule paire de diarques, « Parsman le Léopard, fils d'Amazasp', et Mirdat', fils de Derok' », là où il y en a eu deux. En réalité ont régné successivement Parsman le Bienfaisant et Parsman le Léopard (CK I, 2, 16), puis Mirdat' et Rok' (CK I, 2, 17). $3^{\circ} \mathrm{CK}$ I, 2, 27 compte Lev, père de Mirian, comme un des souverains de sa liste.

Le premier et le troisième écart reflètent probablement des interventions volontaires du rédacteur de C3E. Celui-ci s'éloigne délibérément de sa source pour exprimer son point de vue personnel. Azo (et non Azon) n'est pas un tyran étranger, mais un souverain légitime, un vrai Kartvèle, fils du roi d'Arian Kartli. De même Mirian n'est pas le fils de K'asre, un Sassanide qui se serait imposé par la force. C'est 
simplement le successeur de son père. De cette façon, la christianisation du Kartli est le fruit d'un processus rectiligne et continu, ménagé par la Providence dès la fondation du royaume, sous l'égide d'Alexandre, dont Constantin est le véritable héritier. Dans ce processus endogène, il n'y a pas de place pour les intrus.

Au contraire, le deuxième écart révèle une omission de Leont'i. Elle est si grave que le texte authentique (connu de $\mathrm{CK}$ ) n'est pas restituable à partir de VK. S'il n'avait eu que Leont'i sous les yeux, l'auteur de C3E aurait immanquablement reproduit cette faute. Le fait qu'il nous livre la leçon correcte montre qu'il travaillait à partir d'un autre modèle. Ce modèle, source commune à CK et à VK, pourrait remonter à une tradition orale, antérieure à l'invention de l'alphabet géorgien. Il est hors de doute que les listes de monarques font partie de l'oralité caucasienne.

Alors que C3E ne dépend pas de Leont'i, il est certain que celui-ci s'inspire du Dossier sur Nino, non seulement dans son second livre sur la vie de la sainte, mais aussi pour certains développements de la vie des rois. Par exemple, Leont'i observe que le Christ naquit la première année du règne d'Aderk' sur le Kartli et l'Egrisi : "Quand les Mages entrèrent dans Jérusalem, la nouvelle parvint à Mcxeta que les Perses avaient capturé la ville. Alors les juifs qui étaient à Mcxeta se mirent à pleurer et à se lamenter ». La fausse nouvelle est bientôt démentie. Trente ans plus tard, Elioz, juif de Mcxeta, est convoqué par le Sanhedrin pour participer au jugement de Jésus. Il rapporte en Géorgie la sainte tunique du Sauveur. Cette tradition fait manifestement écho au témoignage de Sidonie, fille d'Abiatar, descendant d'Elioz (CK VII). C3E y fait aussi brièvement allusion (CK I, 6).

En évoquant l'époque reculée des rois païens, le narrateur de $\mathrm{C} 3 \mathrm{E}$ se garde bien d'éclairer les ténèbres. Au contraire, il décrit l'aggravation de l'idolâtrie de règne en règne. Quant aux ombres de l'Ancienne Alliance, dévoilées par la prédication de sainte Nino, il les laisse pressentir d'une façon énigmatique dans l'« Histoire primitive du Kartli » (CK I, 1), qui précède la « Liste des rois païens » (CK I, 2). Commençons par traduire ce texte difficile, tel que nous le comprenons.

Tout d'abord, quand le roi Alexandre eut mis en fuite les tribus des fils de Lot et les eut repoussées dans la terre de K'edar, il vit les tribus féroces des Bun-Turks, qui étaient implantées sur le cours du Mt'k'uari, de part et d'autre, en quatre villes avec leurs villages : la ville de Sark'ine, K'asp'i, Urbnisi et Odzraq'e, ainsi que leurs forteresses, Cixedidi, forteresse de Sark'ine, l'Upliscixe de K'asp'i, la forteresse d'Urbnisi et celle d'Odzraq'e.

Alexandre fut frappé de stupeur en apprenant que les Bun-Turks étaient les tribus des Jébuséens, qui mangeaient tout ce qui est chair, n'avaient pas de tombeaux et dévoraient les morts. Ne pouvant alors les combattre, le roi s'éloigna. Là-dessus arrivèrent les tribus belliqueuses des Huns, issues des Chaldes, et elles demandèrent au roi des Bun-Turks une terre moyennant tribut. Elles s'établirent à Zanav. Et quand elles eurent pris possession de ce qu'elles tenaient moyennant tribut, elles l'appelèrent Xerk'i.

Quelque temps plus tard revint Alexandre, roi de toute la terre. Il anéantit trois des villes et forteresses et frappa les Huns de son glaive. Mais contre la ville de Sark'ine, il combattit douze mois, campant du côté ouest de Sark'ine. Il y planta une vigne et creusa un canal d'irrigation depuis le Ksani. Il préposa des gardiens du canal pour réguler le flux, et l'on appela cet endroit Nast'agisi. Enfin, il prit Sark'ine. D'eux-mêmes les ennemis abandonnèrent la ville et prirent la fuite. 
Le roi Alexandre avait dans son escorte Azo, fils du roi d'Arian-Kartli. Il lui assigna Mcxeta pour résidence, lui fixant pour frontières le Hereti, l'Egris-C'q'ali, le Somxiti et la montagne de Cxol. Puis il partit. Cependant cet Azo se rendit chez son père en Arian-Kartli, d'où il ramena huit Maisons, ainsi que dix Maisons de pères nourriciers. Il s'établit dans le vieux Mcxeta, et ils amenaient avec eux les idoles de Gaci et de Gaïm.

Il y a peu de chance qu'Alexandre ait mis les pieds dans le Caucase, ni personnellement, ni par l'intermédiaire de ses stratèges. Tout au plus, après sa victoire sur les Achéménides, envoya-t-il Ménon, pour occuper les mines d'or de Sper dans les Alpes pontiques. Mais le coup de main échoua et Ménon fut tué. C'est pourquoi ce récit des origines, qui relate l'émergence des Kartvèles (les Géorgiens) sur la scène de l'histoire est moins factuel que mythique. Pour le comprendre, au propre et au figuré, il faut déchiffrer deux niveaux d'énigmes.

Tout d'abord, qui sont les peuples en présence : Bun-Turks, Huns, et Arian-Kartvèles? Gardons-nous de prêter à ces noms symboliques et cryptés une signification géographique ou ethnique trop précise. Comme l'indique l'adjectif bun, qui veut dire " d'origine, natif, autochtone, authentique », les Bun-Turks sont les Turcs aborigènes, qui sont là $a b$ origine, c'est-à-dire les tout premiers habitants du Kartli, leur terre natale depuis des temps immémoriaux, bien longtemps avant l'arrivée des Kartvèles, qui se considèrent pourtant par la suite comme des autochtones.

Où donc les Kartvèles demeuraient-ils avant d'habiter la Géorgie? Notre texte répond à cette question par le nom d'une terre inconnue, d'une localisation très incertaine, l'Arian-Kartli, ce qui veut dire littéralement « la Géorgie iranienne ». Inutile d'observer qu'aucune province iranienne n'a jamais porté un tel nom. Pour comprendre, il faut partir de l'opposition Bun-Turks vs Arian-Kartli, et s'interroger sur la signification culturelle qu'elle pouvait avoir dans la mentalité des chrétiens de Géorgie, du V $\mathrm{V}^{\mathrm{e}}$ au VII ${ }^{\mathrm{e}}$ siècle.

Les liens entre les Géorgiens et les Iraniens remontent au moins aux origines de l'Empire achéménide, et l'Iran a toujours été perçu comme un parangon de civilisation. Rien que d'après son nom, on comprend tout de suite que la société des Arian-Kartvèles devait être très raffinée, autant que celle des Iraniens eux-mêmes à l'apogée de leur gloire et de leur puissance. Au contraire les ethnies turciques, que les Géorgiens peuvent connaître à la même époque, par exemple les Huns et les Khazars, même plus ou moins sédentarisés dans le Nord du Caucase ou sur le rivage occidental de la mer Caspienne, incarnent la barbarie des steppes, prédatrice des grands empires du Proche et du Moyen-Orient.

C'est bien ainsi que les présente notre texte. Par leur nécrophagie, qui horrifie Alexandre, les Bun-Turks se ravalent au plus bas niveau des Barbares. C'est pourquoi Alexandre détruit leurs villes et les oblige à quitter le pays. Quant aux Huns, c'est un peuple belliqueux difficilement contrôlable, mais éventuellement amendable. Après tout, ils acceptent de payer un tribut pour les terres qu'ils occupent; ils s'abstiennent d'arracher les vignes et de détruire les canaux d'irrigation construits pendant le siège de Sark'ine.

C'est peut-être à leur intention qu'Azo, à qui Alexandre a confié le Kartli, après l'expulsion des Bun-Turks, ramène de chez son père dix familles de «pères nourriciers », probablement des gens d'Arian-Kartli, ses compatriotes. Au Caucase, dit-on, 
« le lait va aussi loin que le sang ». Si donc des familles d'Arian-Kartvèles adoptent et nourrissent des enfants d'autres ethnies, ces derniers deviendront les frères de lait des enfants kartvèles. On voit donc s'esquisser un rite de fraternisation entre les Huns de Zanav et les Arian-Kartvèles.

Quant aux huit autres familles, ramenées par Azo, elles ne semblent pas destinées à se mélanger aux ethnies locales, mais elles sont peut-être à l'origine des huit eristav, c'est-à-dire les grands princes kartvèles chargés d'assister le roi dans la défense et dans le gouvernement des provinces.

Cependant il nous faut déchiffrer aussi un second degré de cryptage, dont la clef est surtout biblique et dont la signification est prophétique. Qui sont les fils de Lot et les Jébuséens? Quel rapport ont-ils avec le Kartli et que symbolise Zanav, la ville où se sont établis les Huns?

L'ethnonyme des Jébuséens nous transporte d'un coup du Caucase à Jérusalem et nous fait revivre un moment décisif de l'histoire des Hébreux : la prise de Jérusalem et de la Montagne de Sion par David.

David, avec tout Israël, marcha sur Jérusalem, c'est-à-dire Jébus. Là étaient les Jébuséens, habitants du pays. Les habitants de Jébus dirent à David : «Tu n'entreras pas ici ! » Mais David s'empara de la forteresse de Sion, c'est-à-dire la Cité de David.

En identifiant les Bun-Turcs aux Jébuséens, l'auteur de C3E fait de Sark'ine, toute proche du futur site de Mcxeta, une nouvelle Jérusalem. Il suggère que les Kartvèles s'y sont installés aussi légitimement que les Hébreux dans la Cité de David. À vrai dire, en son temps, l'analogie avait sûrement échappé aux principaux intéressés, y compris au roi Alexandre. Mais l'exégèse typologique de l'Écriture sainte exerce les chroniqueurs chrétiens à prêter aux faits historiques ou supposés tels une signification prophétique dont on ne s'avise qu'à plusieurs siècles d'intervalle. À ce compte, Alexandre, tout païen qu'il est, peut devenir l'ombre projetée de David, l'Oint du Seigneur.

On objectera que la date où nous situons la rédaction de $\mathrm{C} 3 \mathrm{E}$, vers 639, est antérieure aux prétentions des Bagrat'ioni à une royauté davidique. En effet, celles-ci ne sont pas explicitement attestées avant le principat d'Ašot (mort en 830), curopalate du T'ao K'lardžeti, tel qu'il est évoqué dans la Vie de Grigol Xandzteli. Le Dossier sur Nino est d'ailleurs encore plus tardif.

Néanmoins, l'irruption des Jébuséens au début de C3E suggère que la prise de Sark'ine par Alexandre, comme jadis David avait conquis la Montagne de Sion, annonçait que la métropole des Kartvèles était appelée à devenir une nouvelle Jérusalem. Comment pouvait-il en être autrement, dès lors que la sainte tunique du Christ y fut rapportée depuis la Ville Sainte (CK I, 6)? De plus, dans les dernières décennies du $\mathrm{V}^{\mathrm{e}}$ siècle, le Lectionnaire hagiopolitain fut traduit en géorgien et les églises de Mcxeta furent disposées dans des positions homologues aux différentes stations de la liturgie processionnelle en usage pour la Pâque. Le même dispositif est aussi adopté en Arménie.

Ce transfert, dans le Caucase, des grâces salutaires de la Terre Sainte n'aurait pu se réaliser sans la migration et le concours des juifs de Géorgie. Le roi Mirian et sainte Nino y insistent expressément (CK I, 6-7). En dehors de Mcxeta (CK IV) et de plusieurs autres cités, comme Bodi, K'odisc'q'aro, Sobk'anani et Urbnisi, les juifs 
étaient aussi nombreux à Zanav, où les Bun-Turks avaient jadis établi les Huns. Pardelà les données matérielles et documentaires historiquement vérifiables, s'esquisse une sorte de mythologie des origines. On entrevoit ainsi, entre les âges primordiaux et historiques du Kartli, deux changements de population : les Bun-Turks s'effacent devant les Kartvèles et les Huns devant les juifs. Ainsi, se trouvent mises côte à côte les deux nations appelées à réaliser les plans de la Providence : juifs et Géorgiens.

La mention des fils de Lot, chassés par Alexandre dans la terre de K'edar, serait sûrement moins énigmatique, si nous étions sûrs du sens de ce dernier toponyme. La Bible nous apprend que K'edar est le pays du deuxième fils d'Ismaël, chez les Arabes. Mais le rédacteur de C3E l'entendait peut-être autrement. En effet, on lit dans le Psautier : "Malheureux que je suis de séjourner dans Mesek, de demeurer sous les tentes de K'edar ». Cette fois-ci K'edar est associé à Mesek ou à Meshech, qui pourrait évoquer le Meskheti, pays des Meskhes, population proche des Karvèles.

À l'intérieur même de notre chronique (CK X), sainte Nino, exorcisant les cohortes de démons qui entravent la mise en place de la "Colonne Vivante ", leur ordonne : «Repartez pour les ténèbres du nord, les montagnes de K'edar!». Dans la conception archaïque du monde, la chaîne du Grand Caucase marque la limite septentrionale des terres, juste au bord de l'Océan qui les entoure. On dirait presque que la racine sémitique $k d r$ est superposable au géorgien $\check{c} r d$-il « ombre, ténèbres », črd-il-o « nord ». C'est pourquoi la Géorgie est souvent qualifiée de « terre du nord ».

De même que Lot, fuyant le soufre et le feu du ciel projetés sur Sodome et Gomorrhe, se réfugie au sommet d'une montagne et se terre dans une grotte sombre, où ses deux filles lui donnent une descendance masculine, Alexandre chasse devant lui des hordes épouvantées et pénètre dans le «pays de l'ombre »-épithète poétique désignant ici le Kartli -, où il est horrifié par les mœurs des Turcs aborigènes.

Les populations que le chroniqueur nomme «tribus des fils de Lot » ne font sûrement pas partie de ces sauvages, car Lot et sa descendance, les Moabites, ne sont pas des réprouvés. Leur parenté avec Abraham leur vaut la protection de l'Éternel. Ils font plutôt figure de précurseurs des juifs du Kartli, envoyés par la Providence pour instiller la foi chez les Kartvèles.

Ainsi, quoique la «Liste des rois païens » forme une composante narrative, bien antérieure à $\mathrm{C} 3 \mathrm{E}$, et remonte sans doute à l'oralité, le rédacteur du texte la présente d'une façon qui prépare déjà la suite de son récit : l'arrivée des juifs au Kartli, la suppression des idoles et la christianisation du pays.

Bien que l' " Histoire primitive du Kartli », qui sert de prologue à la Liste, intègre des traditions orales sur l'ancien paganisme, elle esquisse subtilement, à l'aide de noms bibliques, une interprétation typologique du peuplement de la Géorgie jusqu'à la conversion : les Kartvèles succèdent aux Bun-Turcs, les juifs aux Khazars, et les chrétiens aux juifs. Contrairement à ce qu'on pourrait croire, la Liste royale de CK n'est pas tirée de Leont'i Mroveli, mais d'une source commune aux deux œuvres. Par conséquent, il n'y a pas de raison de supposer que C3E soit de beaucoup ultérieure au dernier fait datable qu'elle mentionne : la victoire d'Héraclius sur les Perses en 628.

En revanche, le Dossier sur Nino peut être daté du début du IX ${ }^{\mathrm{e}}$ siècle d'après la liste des catholicos incluse dans NSin50. Naturellement ce Dossier a été rédigé tout exprès pour accentuer la dimension prophétique de $\mathrm{C} 3 \mathrm{E}$ et le rôle primordial des juifs 
de Mcxeta. À cette époque, la figure de David commence à prendre une signification politique dans les prétentions généalogiques des Bagrat'ioni et leurs efforts pour restaurer le royaume géorgien.

Néanmoins, quand s'élabore l'historiographie royale de VK, les commanditaires Bagrat'ioni choisissent d'intégrer à la collection officielle des chroniques, non pas $\mathrm{CK}$ (C3E + Dossier), mais la réécriture de Leont'i Mroveli, chronologiquement plus explicite et plus claire. C'est ainsi que CK a été reléguée dans une couche archaïque et résiduelle de l'historiographie géorgienne. Alors que Brosset a traduit VK, dès le milieu du $\mathrm{XIX}^{\mathrm{e}}$ siècle, d'après l'édition du roi Vaxtang, les quatre témoins de CK actuellement connus n'ont été retrouvés que plus tard. Voilà pourquoi ils n'ont pas encore pleinement dévoilé leur profonde originalité.

II. En quête de la philosophie, des arts libéraux et autres disciplines profanes, que les clercs arméniens nomment "sciences du dehors», les Saints Traducteurs, disciples de Maštoc', inventeur de l'alphabet arménien en 405, et de Sahak, interprète de la Bible en 435, sont devenus au cours des siècles des personnages légendaires, à qui l'on prête de fabuleuses pérégrinations dans les métropoles de l'Orient méditerranéen.

L'un de ces récits romanesques, attribué à David l'Invincible, commentateur supposé arménien de l'Organon, raconte comment le futur philosophe, accompagné de son frère Moïse et de trois autres compagnons, furent délégués par leurs maîtres, Maštoc' et Sahak, auprès de Théodose, qui leur fit bâtir les murailles de Théodosiopolis (Erzurum), place forte qui sépare l'Arménie byzantine, ralliée à l'orthodoxie grecque, de la Persarménie, adepte du miaphysisme arménien.

Pour les récompenser, l'empereur les envoie à Athènes, la «ville des philosophes ", où ils étudient sept ans. L'examen final consiste à commenter les sentences des Sept Sages de la Grèce, gravées sur une colonne par le roi de Rome, Servius Tullius. David s'en acquitte si brillamment qu'il mérite le surnom d'Invincible.

Après la mort de Théodose, l'impie Marcien, dominé par la démoniaque Pulchérie, réunit le Concile de Chalcédoine, qui dénature le dogme (selon les Arméniens), en séparant l'humanité du Christ de sa divinité. David et Moïse revenus d'Athènes à Constantinople, réfutent cette hérésie, en répliquant, en présence de l'empereur, aux arguments de Juvénal, l'indigne patriarche de Jérusalem. Cette réfutation constitue le Livre des Etres, manuel scolaire, par questions et réponses, pour appliquer l'Organon à la christologie. L'un des intérêts de cette fiction est de mettre en scène, d'une façon très vivante les joutes académiques où s'exercent les apprentis philosophes et l'enseignement de la philosophie par le commentaire de sentences ou de courtes maximes résumant la doctrine.

On nous permettra de nous arrêter à ce propos sur le cas d'un texte bien connu, les Définitions d'Hermès Trismégiste à Asclépius (DH), dont la version arménienne remonte probablement à la fin du VI ${ }^{\mathrm{e}}$ ou au début du VII ${ }^{\mathrm{e}}$ siècle, et qui permet, grâce aux circonstances de la tradition manuscrite, d'élucider certains aspects des emprunts arméniens à l'éducation libérale du monde grec, tout en découvrant l'usage pratique et pédagogique des textes.

L'authenticité hermétique du texte ne fait aucun doute. Elle est garantie à la

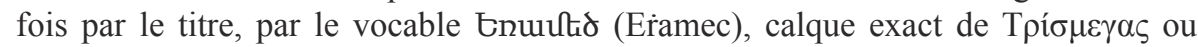




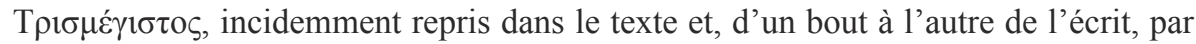
de très nombreux parallèles avec les traités hermétiques grecs.

Deux types d'exercices étaient proposés dans l'antiquité aux disciples d'un maître de rhétorique ou de philosophie : ou bien résumer un cours magistral en brèves sentences faciles à mémoriser; ou bien, en sens inverse, reconstituer l'enseignement complet à partir d'un sommaire de sentences.

Le rédacteur des $\mathrm{DH}$ arméniennes nous livre la matière et le fruit du premier exercice. Il suffit de comparer les öpor / uuhưuip (sahmank') énoncés dans son écrit avec les argumentations des dialogues hermétiques conservés en grec pour comprendre que chaque « définition » résume un enseignement suivi. Malheureusement, aucun exemple de ce type d'enseignement ne nous est aujourd'hui transmis en arménien, car aucun dialogue d'Hermès avec ses disciples ne semble avoir été traduit.

Mais David l'Invincible - disciple arménien et peut-être chrétien du philosophe néoplatonicien Olympiodore - avait meilleure réputation que le sage égyptien. Voilà pourquoi la version arménienne de ses Définitions de la philosophie est devenue dans sa patrie, l'Arménie, le manuel le plus célèbre. Or, chacune des définitions de David fait l'objet d'un commentaire argumenté : c'est donc là un exemple parfait du deuxième type d'exercices scolaires, passer de la sentence à l'enseignement suivi. Rien d'étonnant, d'après le roman des Saints Traducteurs : c'est exactement le genre d'exposé où David avait excellé à Athènes.

Mais il existe encore un autre usage des définitions et des sentences, qu'on pourrait presque qualifier de mystique, car il constitue en tout cas un véritable exercice spirituel. Les écrits hermétiques opposent deux facultés mentales complémentaires : le discours ( $\lambda$ ó o / ban) lié à la parole, à la dialectique, au raisonnement argumentatif, et l'intellect (voṽ / mitk'), qui est une intuition globalisante. Le discours n'en finit plus de décortiquer minutieusement les problèmes; il est incapable de mettre un terme à ses analyses s'il n'est pas relayé, « à un certain point de la route » $(\mathrm{CH}$ IX, 10) par l'intellect, qui lui livre d'un coup la vérité entière et définitive.

DH V, 2.3 (p. 231. 233) observe à ce propos :

C'est dans le silence que l'intellect conçoit le discours; seul le discours issu du silence et de l'intellect est salut. Mais le discours issu du discours n'est qu'une perdition (...). Celui qui parle sans intellect ne dit rien : n'ayant l'intelligence de rien, il n'a pas l'intellect et il parle, car sa parole est foule, et la foule n'a ni intellect ni discours. Le discours doué d'intellect est un don de Dieu.

On peut banaliser le texte en comprenant prosaïquement qu'il faut réfléchir en silence avant de parler.

Mais les disciples d'Hermès les mieux instruits préféraient se projeter à l'intérieur de l'Intellect divin qualifié d'Autogène, parce qu'il est le reflet éternel du Dieu Inengendré, qui n'a pas de conjoint. Cependant il doit lui-même s'unir à sa conjointe Silence pour produire le divin discours Engendré.

Attestée en copte dans L'Ogdoade et l'Ennéade, la Triade divine - Inengendré, Autogène, Engendré - n'est pas explicitement présente dans les $\mathrm{DH}$, mais on en perçoit un écho indirect en arménien dans un fragment: "Hermès, l'illustre philosophe, professe trois Puissances des cieux, très grandes, ineffables, créatrices de 
toutes choses et soutient que c'est là l'unique divinité ». Il est donc assuré, dans l'hermétisme, que le Silence de l'âme, reflet d'une hypostase divine, est indispensable à l'éveil de l'intellect et à la connaissance de Dieu.

Or, rien n'est plus difficile à obtenir que le silence. Car il ne suffit pas de rester muet pour faire taire le discours intérieur. Dès qu'on cherche à se recueillir pour faire silence en soi-même, les pensées importunes accourent en foule, chacune s'empresse de se nommer et de proclamer ses exigences, sans réfléchir. Dans un tel tumulte, l'illumination de l'intellect est impossible.

Mais le sage, qui s'est maintes fois exercé à passer du discours à la sentence et de la sentence au discours, perçoit au moindre signal la totalité du message. Un seul mot suffit à le transporter au-delà du langage, dans la lumière du pur concept. Les pauses silencieuses marquées par les points séparant entre elles les diverses sentences de la gnomologie deviennent pour lui des moments de paix, des pistes d'envol vers les évidences transcendantes qu'il a longuement contemplées lors de ses précédents exercices.

En DH IX, 4, on lit (p. 265) : "Qui se réfléchit en intellect, se connait, et qui se connaît, connaît toute chose ». Cela revient à dire que les trois gnoses - de Dieu, de soi-même et du Tout - sont équivalentes. Mais on a aussi rapproché cet adage d'une des «paroles secrètes » de Jésus dans l'Évangile de Thomas : «Qui se trouve luimême est supérieur au monde ». Comme cet apocryphe, les DH peuvent être tenues pour un recueil de paroles cryptées, volontairement détachées de tout contexte existentiel ou narratif, pour permettre des moments de médiation silencieuse.

"Que celui qui cherche ne cesse de chercher jusqu'à ce qu'il trouve et, quand il trouvera, il sera troublé et, ayant été troublé, il s'émerveillera et il régnera sur le Tout ». L'émerveillement se suffit à lui-même. Il rend muet d'admiration. Il joue un rôle décisif dans l'instauration du silence intérieur, du vide cathartique, indispensable à la connaissance de Dieu. Ce lien est souligné en DH IX, $6:$ « Dieu est digne d'adoration, l'homme, d'émerveillement ». C'est à l'homme qu'il convient d'adorer Dieu; mais Dieu fut le premier à s'émerveiller devant le monde qu'il avait créé. Il a fait l'homme à son image pour partager le même émerveillement. S'émerveiller revient donc à partager le regard aimant du Créateur sur le Tout.

On comprend ainsi que, par-delà leur usage purement scolaire, les DH pouvaient aussi donner lieu à des exercices spirituels visant à réchauffer la ferveur de l'intellect pour l'élever vers Dieu. Les échos de la version arménienne qu'on relève chez les auteurs médiévaux confirment que ces orientations mystiques n'ont pas été méconnues.

Le premier pas de l'exercice spirituel est de se libérer des contingences individuelles pour se replacer dans la perspective du Tout. C'est justement par-là que commencent les DH :

Dieu, monde intelligible ; monde : Dieu sensible; homme : monde destructible. Dieu : monde immobile ; ciel : monde mobile; homme : monde raisonnable (...). Deux unités ne font qu'un : Dieu et l'homme à son image. Il y a donc trois mondes en tout (...). Deux sont visibles : le sensible et le monde destructible qu'est l'homme; tandis que l'intelligible, c'est Dieu que voici : il n'est pas visible, mais apparaît dans les choses visibles d'ici-bas. 
Pour comprendre la condition humaine - l'homme mortel et conscient de sa mortalité - il faut concevoir l'être humain comme un "monde ", c'est-à-dire une parure

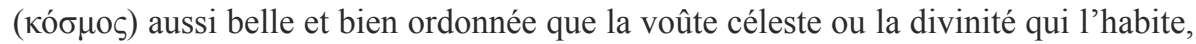
en sorte qu'il y ait une égale dignité entre ces trois êtres et que l'homme compense la fragilité de sa vie par un lien privilégié avec la raison divine universelle.

Il est donc impossible que Dieu ne montre pas à l'homme, " monde raisonnable », plus de sollicitude qu'au monde "sans raison ». C'est bien ainsi que l'entend Ełišê, le chroniqueur arménien du $\mathrm{VI}^{\mathrm{e}}$ siècle : «Et si Dieu prend tel souci de ce monde sans raison, combien plus de l'homme, monde raisonnable! ».

Mais au XI ${ }^{\mathrm{e}}$ siècle, le savant platonicien, Grigor Magistros, assimilant Dieu à l'Intelligible, traite de corruptibles les deux autres mondes :

Méditer la corruption de ces deux êtres, macrocosme et microcosme, surtout s'ils restent extérieurs aux ordres du monde intelligible. Car le monde intelligible s'entend comme l'essence divine d'après Hermès Trismégiste. Quant au microcosme, c'est notre genre humain, et le macrocosme, c'est le monde où nous sommes établis.

Pour s'engager dans la «voie d'immortalité ", il faut avoir foi dans les deux facultés dont Dieu a doté l'homme, le discours et l'intellect. C'est ce qu'a retenu, vers 670, le mathématicien Anania Širakac'i, auteur d'une Cosmographie qui s'efforce de concilier la révélation chrétienne et la science antique : « Pour le discours, il n'est rien d'inexprimable, et pour l'intellect, rien d'inaccessible ».

Fort d'une telle assurance, l'homme peut entreprendre l'explication de l'univers dans son ensemble et, à travers cela, connaître le Créateur :

Généreuse, la nature ici-bas enseigne à tous. Si rien de ce qui est ne te paraît œuvre vaine, tu trouveras l'œuvre et l'artisan. Si cela te semble être un jeu, c'est toi qui seras joué (...) Car tu as le pouvoir de vouloir et de ne pas comprendre, tu as le pouvoir de manquer de foi et de te tromper, et de comprendre le contraire de ce qui est.

Une fois reconnue la place de l'homme, monde raisonnable, au cœur du monde sensible, on peut revenir avec plus d'assurance vers la condition de l'individu, le mystère de la naissance et de la mort : "Mort bien comprise est immortalité; non comprise, elle est mort ». C'est à cette formule que recourt Ełišē pour fustiger la lâcheté d'éventuels apostats, prêts à renier la foi pour échapper au martyre.

Comme on l'a dit justement autrefois : la mort non comprise est mort; la mort comprise est immortalité.

Lecteur d'Ełišē, Grégoire de Narek vivait à une époque où les Arméniens étaient relativement à l'abri des persécutions religieuses zoroastriennes ou musulmanes. C'est pourquoi il applique la même maxime non pas aux renégats occasionnels, mais à tous les chrétiens indécis qui ne croient pas suffisamment à la vie éternelle :

Comme je ne sais quel sophiste profane / jugea bon de nommer la mort mauvaise quand on n'en connaît pas la cause, / je témoigne en ces termes à mon tour. / À la façon des bêtes sans conscience, / nous courons au trépas et n'en sommes pas interdits, / on nous enterre et nous ne voulons pas nous humilier.

Si la mort est pur néant, elle ne peut constituer un objet de pensée. Mais si, comme l'enseigne la foi, elle est un passage vers une autre vie, comment se figurer cette 
transition? Faute de trouver réponse à cette question dans le Nouveau Testament, il semble que les Arméniens aient interrogé Hermès Trismégiste.

De même que tu es sorti du ventre, de même aussi tu sortiras de ce corps ; de même que tu n'entreras plus dans le ventre, de même aussi tu n'entreras plus dans ce corps matériel. De même que, dans le ventre, tu ne savais pas les choses de ce monde, de même aussi, sorti du corps, tu ne reconnaîtras pas les êtres extérieurs au corps. De même que, sortant du ventre, tu ne te souviens en rien des choses du ventre, de même aussi, sortant de ce corps, tu ne te souviendras de rien de ce qui est à lui.

On comparera cet enchaînement d'analogies au Yačaxapatum, recueil homilétique $\mathrm{du} \mathrm{VI}^{\mathrm{e}}$ siècle, attribué tantôt à Grégoire l'Illuminateur, et tantôt à Maštoc' .

De même que les enfants, dans le ventre des mères sont enfouis dans le sang et dans les ténèbres et ne connaissent pas la clarté de cette terre (...), de même aussi il ne nous appartient pas de connaître la vie future.

Comme le sage païen, le prédicateur chrétien compare la naissance, où le corps de l'enfant sort du corps de la mère, à la mort, où l'âme sort du corps à qui elle donnait la vie. Dans les deux cas, ce changement s'accompagne d'ignorance ou d'oubli. Pour Hermès Trismégiste, de même que l'enfant oublie sa vie utérine après la naissance, de même l'âme, sortie du corps, oublie toute vie corporelle. Pour le Yačaxapatum, l'embryon dans le ventre ignore la lumière du monde, et l'âme, dans le corps, est incapable de se figurer ce qui l'attend après la mort.

On comprend bien que le prédicateur chrétien, croyant en la résurrection de la chair, n'ait pas retenu la phrase de Trismégiste «tu n'entreras plus dans ce corps matériel ». En outre, le croyant, initié aux mystères de la foi, est censé reconnaître les êtres célestes auxquels il sera confronté après sa mort. L'analogie hermétique ne pouvait donc être reprise intégralement. Elle devait même être fortement amendée.

Ces détournements chrétiens de la sagesse «du dehors » sont particulièrement instructifs. Ils nous révèlent la double visée des emprunts arméniens à la littérature profane. D'un côté les textes sont utilisés comme moyens d'enseignement, pour un usage pratique : offrir matière à des exercices rhétoriques ou dialectiques purement formels. Mais d'autre part, les païens, si pervertis qu'ils fussent, n'ont pas entièrement effacé de leur conscience la mémoire de Dieu. Car « ce qu'on peut connaître de Dieu est devenu évident pour les hommes. Dieu lui-même l'a rendu tel, puisque ses perfections invisibles, sa puissance éternelle et sa divinité se voient comme à l'œil nu depuis la création du monde, quand on les considère dans ses ouvrages. Aussi les hommes sont-ils inexcusables parce que, tout en connaissant Dieu, ils ne lui ont pas rendu la gloire qui lui était due ».

Au début de sa Cosmographie, Anania Širakac'i se réclame de l'Épître aux Romains pour reprendre, d'un point de vue chrétien, les théories cosmologiques des « bons philosophes », qui n'ont pas été complètement aveuglés par l'idolâtrie et peuvent encore offrir des lumières utiles à l'acquisition de la vérité. Sans aucun doute, aux yeux de ses copistes et de ses lecteurs, Hermès Trismégiste faisait partie de ces sages christiano-compatibles.

$\mathrm{Au}$ contraire de certaines autres langues anciennes du Proche-Orient chrétien, comme le grec et le copte, l'arménien nous livre assez peu d'informations sur les 
écrits gnostiques des trois premiers siècles. Cela tient sans doute à des raisons chronologiques : l'alphabet de Maštoc' est apparu à une époque où le combat contre l'hérésie avait déjà porté ses fruits. De plus, on a censuré la littérature hérésiologique pour éviter la contagion, de crainte que le remède ne devienne aussi redoutable que le mal.

En tout cas, on observe que le traité d'Eznik Contre les sectes (vers 435) ne livre que des informations indirectes et marginales, sur Marcion, Bardesane et Valentin. La version arménienne des cinq livres d'Irénée Contre les hérésies nous est parvenue très incomplètement. On ne dispose aujourd'hui, en continu, que des livres IV et V sur l'Évangile et l'Apostolicon. On peut donc supposer que la partie la plus polémique de l'œuvre, et notamment le livre I, décrivant en détail les systèmes gnostiques, a été laissée de côté, soit parce que le débat n'était plus d'actualité, soit parce que cette érudition hérésiologique risquait de revigorer des hydres encore menaçantes, comme la doctrine des Barbéliotes, qui se cachent peut-être sous les « Barbariens » de Koriwn.

Dans ces conditions, les DH constituent, en langue arménienne, l'un des rares reflets d'une pensée authentiquement gnostique. La sérénité de l'exposé n'est troublée par aucune polémique. En effet, l'hermétisme est une gnose optimiste, qui s'efforce de réduire toute forme de dualisme et attribue la création du monde au Dieu unique, qui se manifeste dans la Triade, Inengendré, Autogène, Engendré. Une telle " gnose » rejoignait les orientations spirituelles d'Évagre le Pontique, traduit en arménien au $\mathrm{V}^{\mathrm{e}}$ siècle, et remis en honneur à Narekavank' au $\mathrm{X}^{\mathrm{e}}$ siècle. 